\title{
Cdc42 deficiency induces podocyte apoptosis by inhibiting the Nwasp/stress fibers/YAP pathway
}

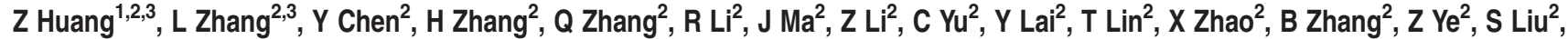 \\ W Wang ${ }^{2}$, X Liang $^{2}$, R Liao ${ }^{2}$ and W Shi*
}

Podocyte apoptosis is a major mechanism that leads to proteinuria in many chronic kidney diseases. However, the concert mechanisms that cause podocyte apoptosis in these kidney diseases are not fully understood. The Rho family of small GTPases has been shown to be required in maintaining podocyte structure and function. Recent studies have indicated that podocytespecific deletion of Cdc42 in vivo, but not of RhoA or Rac1, leads to congenital nephrotic syndrome and glomerulosclerosis. However, the underlying cellular events in podocyte controlled by Cdc42 remain unclear. Here, we assessed the cellular mechanisms by which Cdc42 regulates podocyte apoptosis. We found that the expression of Cdc42 and its activity were significantly decreased in high glucose-, lipopolysaccharide- or adriamycin-injured podocytes. Reduced Cdc42 expression in vitro and in vivo by small interfering RNA and selective Cdc42 inhibitor ML-141, respectively, caused podocyte apoptosis and proteinuria. Our results further demonstrated that insufficient Cdc42 or Nwasp, its downstream effector, could decrease the mRNA and protein expression of YAP, which had been regarded as an anti-apoptosis protein in podocyte. Moreover, our data indicated that the loss of stress fibers caused by Cdc42/Nwasp deficiency also decreased Yes-associated protein (YAP) mRNA and protein expression, and induced podocyte apoptosis. Podocyte apoptosis induced by Cdc42/Nwasp/stress fiber deficiency was significantly inhibited by overexpressing-active YAP. Thus, the Cdc42/Nwasp/stress fibers/YAP signal pathway may potentially play an important role in regulating podocyte apoptosis. Maintaining necessary $\mathrm{Cdc} 42$ would be one potent way to prevent proteinuria kidney diseases.

Cell Death and Disease (2016) 7, e2142; doi:10.1038/cddis.2016.51; published online 17 March 2016

Podocytes are highly differentiated cells with complex actin cytoskeletal architecture that play a key role in maintaining the integrity of glomerular filtration barrier. Therefore, loss of podocyte will surely disturb the function of glomeruli. Apoptosis is one of the main reasons that causes loss of podocyte and sequentially induces proteinuria. Accumulating evidence show that podocyte apoptosis is one of the most important mechanisms in the pathogenesis of many kidney diseases, such as chronic kidney diseases, ${ }^{1}$ diabetic nephropathy, ${ }^{2,3}$ focal segmental glomerular sclerosis, ${ }^{4}$ et al. Thus, preventing podocyte apoptosis will be a promising therapeutic target for treating these kidney diseases. However, the concert mechanisms that cause podocyte apoptosis in these kidney diseases are still far from being fully understood.

Rho family small GTPases RhoA, Rac1, and Cdc42 are the three most extensively studied prototypes, and are known powerful regulators of actin cytoskeletal dynamics, cell adhesive interactions, motility, or cell polarity. ${ }^{5}$ However, these Rho-GTPases also control other important cellular functions, such as gene expression, proliferation and apoptosis. ${ }^{5,6} \mathrm{~A}$ large body of data implicates RhoA, Rac1, and Cdc42 in the pathogenesis of disease processes in the kidney including glomerular diseases. ${ }^{6-10}$ Recent studies also indicated that podocyte-specific deletion of Cdc42 in vivo, but not of RhoA or Rac1, leads to congenital nephrotic syndrome, podocyte foot process effacement and glomerulosclerosis. ${ }^{11,12}$ However, whether Cdc42 is involved in podocyte apoptosis remains unknown. Meanwhile, further studies will also be required to unravel the detailed downstream signaling cascades of Cdc42 in podocytes and the exact mechanisms leading to podocyte apoptosis and proteinuria.

Yes-associated protein (YAP) is a major downstream cascade of the Hippo pathway which controls the expression of genes that promote cell proliferation and inhibits apoptosis. ${ }^{13,14}$ Under normal condition, dephosphorylated YAP localizes in the nucleus and functions as a transcriptional co-activator that mainly through interacting with TEA domain family member family transcriptional factors to induce target gene expression. ${ }^{15}$ YAP phosphorylation promotes its cytoplasmic sequestration and inactivation. ${ }^{16,17}$ Recent studies have indicated that YAP is an anti-apoptotic molecule in podocyte, and podocyte-specific deletion of YAP leads to proteinuria kidney diseases. ${ }^{18,19}$ Moreover, loss of stress fiber

\footnotetext{
${ }^{1}$ Southern Medical University, Guangzhou, Guangdong, China and ${ }^{2}$ Department of Nephrology, Guangdong General Hospital, Guangdong Academy of Medical Sciences, Guangzhou, China

*Corresponding author: W Shi, Department of Nephrology, Guangdong General Hospital, Guangdong Academy of Medical Sciences, 106 Zhongshan No. 2 Road, Guangzhou 510080, China. Tel: +86-13822182116; Fax: +86-20-83827812-62027; E-mail: shiweigd@126.com

${ }^{3}$ These authors contributed equally to this work.

Abbreviations: ADR, adriamycin; HG, high glucose; LPS, lipopolysaccharide; Nwasp, neuronal Wiskott-Aldrich syndrome protein; siRNA, small interfering RNA; YAP, Yes-associated protein

Received 29.10.15; revised 06.2.16; accepted 15.2.16
} 
caused by insufficient Cdc42 can result in reducing transcriptional activity and nuclear localization of YAP. ${ }^{20,21}$ Therefore, it is likely that Cdc42 deficiency may induce podocyte apoptosis by reducing nuclear YAP.

In this study, we demonstrate that the loss of Cdc42 in vitro and in vivo by small interfering RNA (siRNA) and selective inhibitor ML-141, respectively, caused podocyte apoptosis and proteinuria, accompanied with increased pro-apoptotic Bax and decreased anti-apoptotic Bcl-2 gene and protein expression. Our results further demonstrated that insufficient Cdc42 or neuronal Wiskott-Aldrich syndrome protein (Nwasp), its downstream effector, could decrease the mRNA and protein expression of YAP, which had been regarded as an anti-apoptosis protein in podocyte. Moreover, our data indicated that the loss of stress fibers caused by Cdc42/Nwasp deficiency decreased YAP mRNA and protein expression, and induced podocyte apoptosis. Cell apoptosis induced by Cdc42/Nwasp/stress fiber deficiency was significantly inhibited in podocytes with overexpressed-active YAP. Therefore, the Cdc42/Nwasp/stress fibers/YAP signal pathway may potentially play an important role in regulating podocyte apoptosis. Maintaining necessary Cdc42 would be one potent way to prevent proteinuria kidney diseases.

\section{Results}

Apoptosis was significantly increased in high glucose (HG)-, lipopolysaccharide (LPS)- or adriamycin (ADR)injured podocytes in vitro and vivo. To confirm podocyte apoptosis in $\mathrm{db} / \mathrm{db}$ mice or the mouse model of LPS or ADR-induced proteinuria and podocytopathy, podocyte apoptosis in glomeruli was examined by immunostaining with antiWT-1 antibody, a podocyte-specific marker, and TUNEL. As shown in Figures 1a (on top) and b, there was a marked reduction in glomerular WT-1-positive cells in glomeruli of $\mathrm{db} /$ $\mathrm{db}$ mice, LPS mice or ADR mice relative to control mice. TUNEL staining (Figures 1a (below) and c) demonstrated that podocyte apoptosis was obviously increased in $\mathrm{db} / \mathrm{db}$ mice, LPS mice or ADR mice compared to control mice $(0.30 \pm 0.11$ versus $2.05 \pm 0.14,1.95 \pm 0.09$ and $2.85 \pm 0.13$, respectively, $n=3, P<0.05)$. Consistent with the results above, urinary albumin excretion $(\mu \mathrm{g} / \mathrm{mg}$ creatinine), the parameters of proteinuria and podocytopathy, were also remarkably increased in $\mathrm{db} / \mathrm{db}$ mice, LPS mice or ADR mice as compared with that in control mice (Figures 1d-f).

In addition, cell apoptosis was also further evaluated in vitro-cultured podocytes treated with HG, LPS or ADR. As shown in Figures $1 \mathrm{~g}-\mathrm{l}$, cell apoptosis rates were $9.53 \pm 0.15$, $9.93 \pm 2.64,18.43 \pm 3.02,16.59 \pm 1.97,18.89 \pm 1.00 \%$ in control, mannitol (MA), HG, LPS and the ADR group, respectively. Consistent with the results above, the mRNA and protein expression of Bax, a well-recognized indicator of apoptosis were significantly increased in HG, LPS and ADR-treated podocytes comparing to normal controls (Figures 1m-0). However, Bcl-2, the indicator of antiapoptosis was significantly decreased in HG, LPS and ADR-treated podocytes (Figures 1m-0). These data indicated that cell apoptosis was significantly increased in HG, LPS or ADR-injured podocytes in vitro and in vivo.
Cdc42 expression and activity were decreased in injured podocytes in vitro and in vivo. Cdc42 deletion results in massive neonatal proteinuria, kidney failure, and associated death within 2 weeks of birth, podocyte-specific RhoA and Rac1 deletion mutants are conspicuously healthy. Based on this, we explored that the Cdc42 expression and activity in injured podocyte in vitro and in vivo. As shown in Figures 2a-c, the mRNA and total protein expression of Cdc42 were significantly decreased in injured podocyte induced by HG (30 mM) or LPS or ADR comparing to normal controls. Similarly, Cdc42 activity was also obviously decreased in HG or LPS or ADR-treated podocytes. The percentages of Cdc42 activity were 100, 98.85 \pm 1.19 , $55.52 \pm 15.29,67.11 \pm 9.19,50.34 \pm 16.97 \%$ in control, MA, HG, LPS and the ADR group, respectively (Figure 2d).

Next, we investigated Cdc42 expression in LPS or ADR-treated mice, which the former was well-recognized proteinuric mouse model, ${ }^{22,23}$ the latter was a well-known experimental model of human proteinuria disease resemble to FSGS. ${ }^{4,24}$ Immunofluorescent staining exhibited Cdc42 expression was decreased in glomerular podocyte of LPS or ADR-injected mice, comparing with vehicle control mice, respectively (Figure 2e). Using immunofluorescent staining, we also investigated the expression of Cdc42 in glomerular podocytes of patients with nephropathy (DN) or focal segmental glomerulosclerosis (FSGS). Consistent with the results from mice, in patients with DN or FSGS, the protein expression of Cdc42 was also markedly decreased in glomerular podocytes (Figure 2f).

Loss of Cdc42 caused podocyte apoptosis and proteinuria. To further explore the role of decreased Cdc42 in podocyte, we studied the proteinuria and podocyte apoptosis in vitro and in vivo. As shown in Figures $3 a-f$, apoptosis was markedly increased in vitro-cultured Cdc42 knockdown podocytes compare to siCon. We also investigated the mRNA and protein expression of $\mathrm{Bax}$ and $\mathrm{Bcl} 2$, which are two well-recognized indicators of apoptosis. ${ }^{1}$ As shown in Figures $3 g-i$, the mRNA and protein analysis of apoptosis signals Bax showed significantly increased expression in Cdc42-silenced podocytes, as apoptosis negative-related gene and protein Bcl-2 was inversely declined.

In addition, we surveyed podocytes in glomeruli by immunostaining with anti-WT-1 antibody, a podocyte-specific marker. As shown in Figures 3k and I, there was a marked reduction in glomerular WT-1-positive cells in glomeruli of mice treated with Cdc42-specific inhibitor ML-141, Furthermore, measurement of apoptosis cells by TUNEL (Figure 3k, below, $3 \mathrm{M})$ staining showed that the number of apoptosis podocytes in glomerular was increased in mice treated with $\mathrm{ML}-141$ comparing to the control mice $(0.25 \pm 0.10$ versus $2.05 \pm 0.11$, $n=3, P<0.05)$. Urinary albumin excretion was also significantly increased in ML-141-treated mice as compared with control mice (Figure 3j).

Nwasp and YAP expression were decreased in injured podocytes. To further explore the underlying mechanism of Cdc42-induced podocyte apoptosis, we next investigated the expression of its downstream effector Nwasp and transcriptional co-activator YAP in HG or LPS or 
a

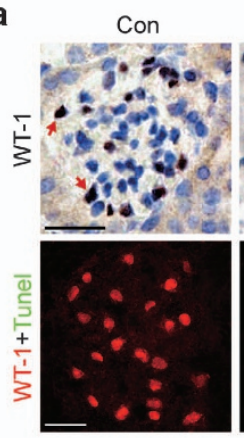

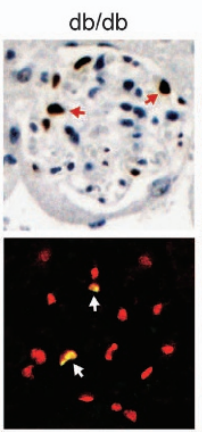

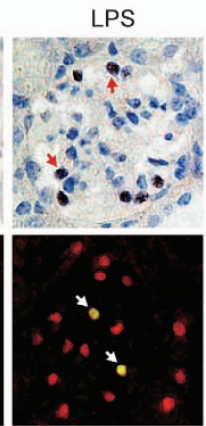

$A D R$



b

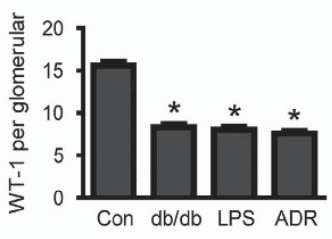

c

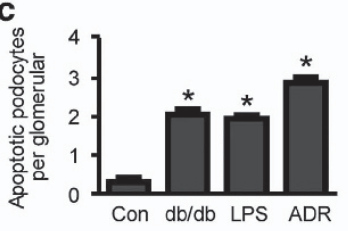



e
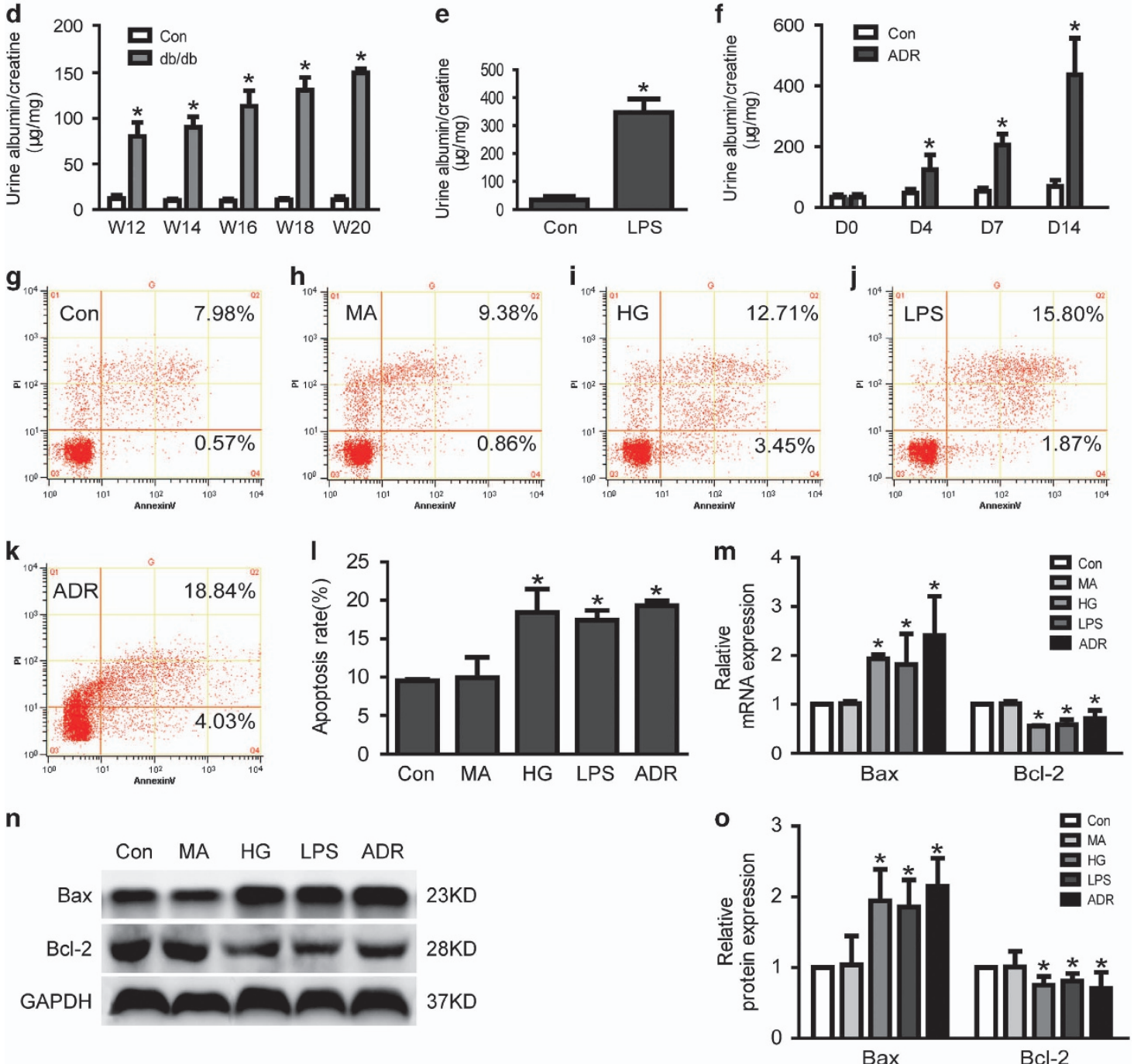

Figure 1 Apoptosis was increased in HG, LPS or ADR-injured podocytes in vitro and in vivo. (a) On top: typical images of immunochemistry stained (WT-1-positive cells, brown) glomeruli from control, db/db, LPS or ADR mice. Below: representative micrographs of dual-color fluorescence staining of kidney glomeruli for WT-1 (red) and TUNEL (green) from control, db/db, LPS or ADR mice. Magnification $\times 400$, scale bar $=25 \mu \mathrm{m}$. (b) Quantification of podocyte markers WT-1-positive cells per glomerular section. The number of WT-1-stained nuclei was obtained from 20 glomeruli per kidney section from five mice per group. (c) Absolute count of the numbers of glomerular cells positive for both TUNEL and WT-1 (white arrows, apoptotic podocyte), data were expressed as the means of apoptotic podocytes from 20 randomly selected glomeruli. ${ }^{*} P<0.05$ versus Con, $n=3$. (d-f) Proteinuria was obviously increased in db/db, LPS or ADR mice compared to control mice. (g-l) Podocytes were stained with Annexin V/PI for flow cytometry analysis. Cell apoptosis rate was significantly increased in HG, LPS or ADR-treated podocytes comparing to normal controls. (m) Bax mRNA expression was increased as Bcl-2 was decreased in HG, LPS or ADR-treated podocytes. (n-0) Bax protein expression was increased as Bcl-2 was decreased in HG, LPS or ADR-treated podocytes. Podocyte apoptosis in MA (as an osmotic control) and the Con group show hardly any difference in all in vitro studies $(\mathbf{g}-\mathbf{0})$. Data were from at least three independent experiments. ${ }^{*} P<0.05$ versus controls 


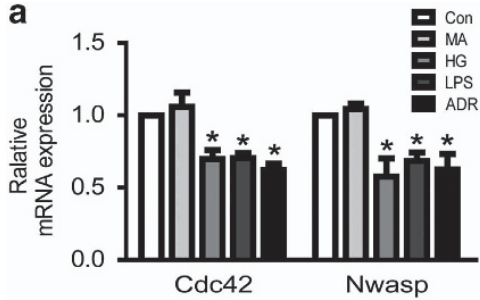

C
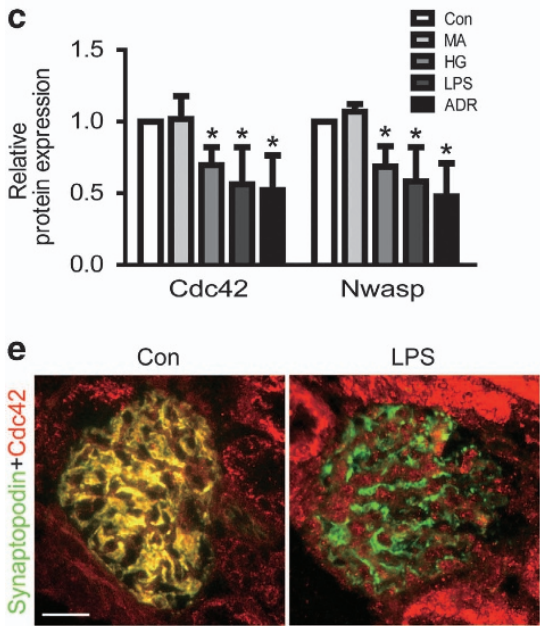

f
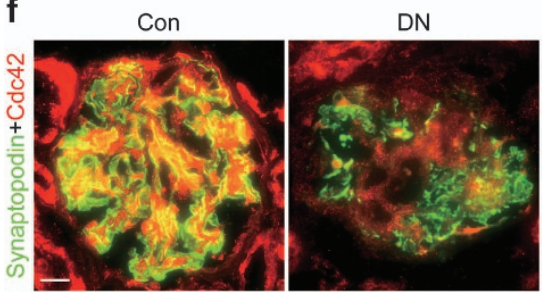

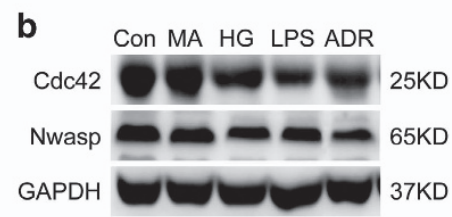

d
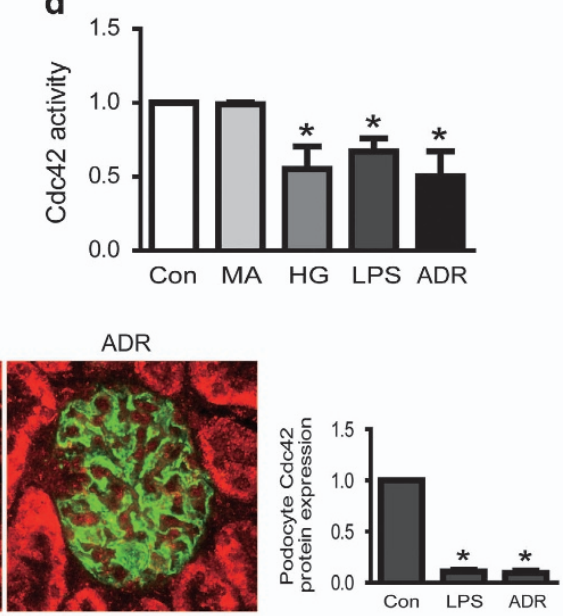

Figure 2 Cdc42 and Nwasp were decreased in injured podocytes. (a) The mRNA expression of Cdc42 and Nwasp were decreased in HG, LPS or ADR-injured podocytes. (b, c) The protein expression of Cdc42 and Nwasp were decreased in HG, LPS or ADR-injured podocytes. (d) Cdc42 activity was decreased in HG, LPS or ADR-injured podocytes. (e) Representative micrographs of dual-color fluorescence staining of kidney glomeruli for Cdc42 (red) and synaptopodin (green) from control, LPS and ADR mice. Magnification $\times 400$, scale bar $=25 \mu \mathrm{m}$. (f) Representative micrographs of dual-color fluorescence staining of kidney glomeruli for Cdc42 (red) and synaptopodin (green) from control, DN and FSGS patients. Magnification $\times 400$, scale bar $=25 \mu \mathrm{m}$. All in vitro studies result in MA (as an osmotic control) and the Con group show hardly any difference (a-d). All data were from at least three independent experiments. ${ }^{*} P<0.05$ versus controls

ADR-injured podocytes. As shown in Figures $2 a-c$, the results of real-time-qPCR and western blot analysis indicated that the mRNA and protein expression of Nwasp were markedly decreased in podocytes treated with HG or LPS or ADR. Results from immunofluorescence and western blotting demonstrated that both nuclear and cytoplasmic YAP protein expression was obviously decreased in injured podocytes in vitro and in vivo (Figures $4 \mathrm{a}, \mathrm{c}-\mathrm{h}$ ). Similar results from PCR to analysis the mRNA expression of YAP were also obtained in HG or LPS or ADR-treated podocytes (Figure 4b). These data indicated that the Nwasp and YAP expression were significantly decreased in HG, LPS or ADR-injured podocytes.

Loss of Nwasp or YAP-induced podocyte apoptosis in vitro. As lost of Cdc42 induced podocyte apoptosis, we investigated whether loss of its downstream effector Nwasp or YAP could induce podocyte apoptosis. As shown in Figures $3 a-f$, cell apoptosis rate was markedly increased in vitro-cultured Nwasp or YAP knockdown podocytes compared with siCon. Consistent with the results above, the mRNA and protein expression of Bax, a wellrecognized indicator of apoptosis were significantly increased in Nwasp or YAP knockdown podocytes comparing to siCon (Figures 3g-i). However, Bcl-2, the indicator of anti-apoptosis was significantly decreased in Nwasp or YAP knockdown podocytes (Figures $3 \mathrm{~g}-\mathrm{i}$ ).

Overexpression of active YAP-alleviated podocyte apoptosis induced by HG, LPS or ADR. To further confirm the role of YAP in podocyte apoptosis, we investigated the effect of overexpression of active YAP in podocyte apoptosis induced by HG, LPS or ADR. Consistent with the results mentioned above, cell apoptosis rate was markedly increased in vitro-cultured podocytes treated with HG, LPS or ADR, respectively (Figures 5b-e). However, after overexpressed-active YAP in vitro podocytes, cell apoptosis induced by $\mathrm{HG}$, LPS or ADR was significantly alleviated 
a

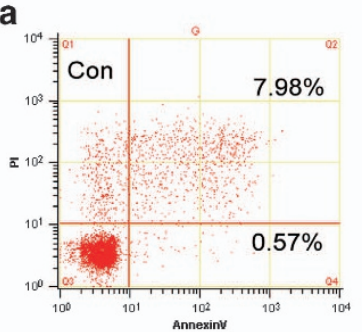

e

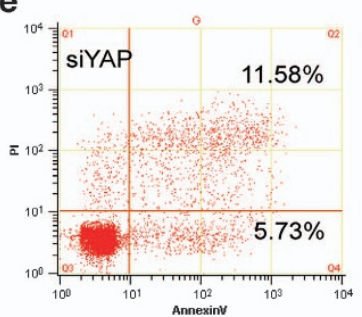

h

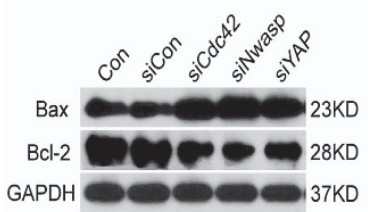

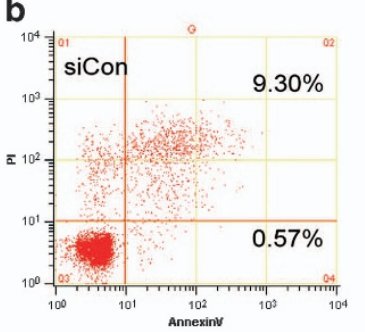

f

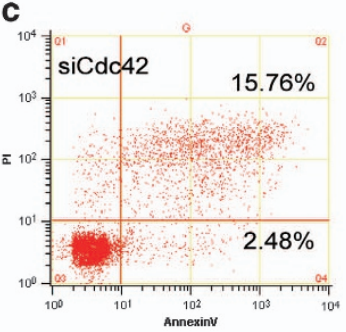

d

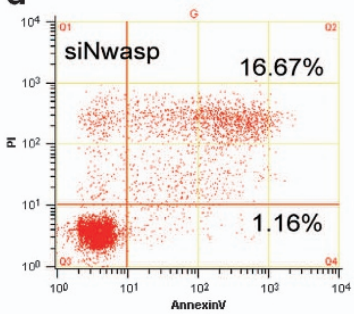

g

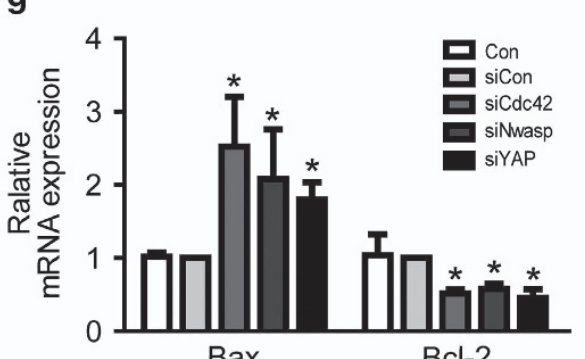

Bax

j

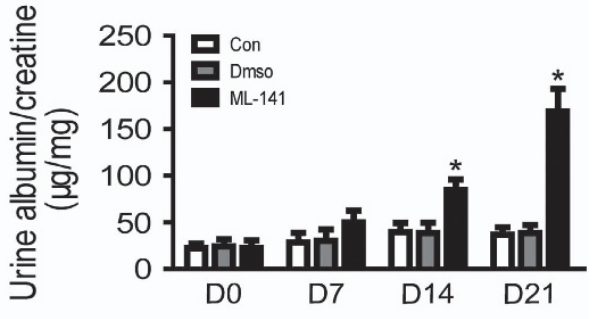

k

Con

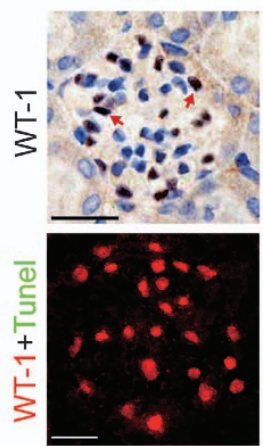

Dmso

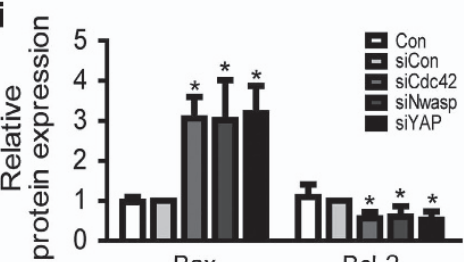

Bax

$\mathrm{Bcl}-2$
$\mathrm{ML}-141$

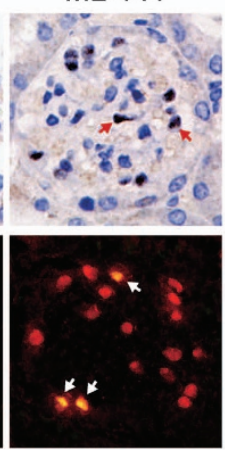

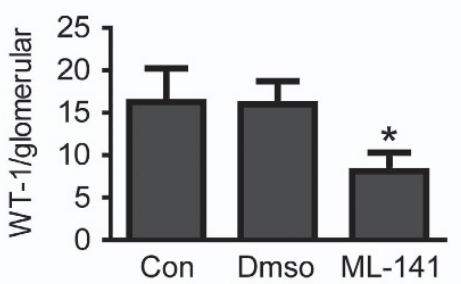

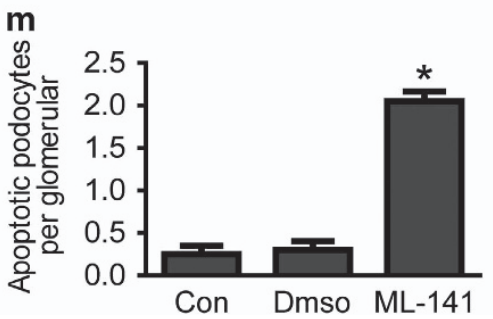

Figure 3 Loss of Cdc42, Nwasp or YAP-induced podocyte apoptosis. (a-f) Podocytes were stained with Annexin V/PI for flow cytometry analysis. Cell apoptosis rate was significantly increased in Cdc42, Nwasp or YAP knockdown podocytes. Con, siCon, siCdc42, siNwasp, siYAP were short for blank control, control siRNA, Cdc42 siRNA, Nwasp siRNA, YAP siRNA, respectively. (g) Compared to siCon, the mRNA expression of Bax was obviously increased as Bcl-2 was obviously decreased in Cdc42, Nwasp or YAP knockdown podocytes. (h-i) Compared to siCon, the protein expression of Bax was obviously increased as Bcl-2 was obviously decreased in Cdc42, Nwasp or YAP knockdown podocytes. (j) A 24h-pooled urine sample was collected in a metabolic cage at day 0, 7, 14, 21 after the first injection of ML-141 for each mouse. Urinary albumin and creatinine were measured using a competitive ELISA. Proteinuria became obvious after injected with ML-141(Cdc42-specific inhibitor) for 14 days. $n=5$. (k) On top: typical images of immunochemistry-stained (red arrows point to WT-1-positive cells) glomeruli from control, Dmso and ML-141 mice. Below: representative micrographs of dual-color fluorescence staining of kidney glomeruli for WT-1 (red) and TUNEL (green) from control, Dmso and ML-141 mice. White arrows point to glomerular cells positive for both WT-1 and TUNEL (apoptotic podocyte), magnification $\times 400$, scale bar $=25 \mu \mathrm{m}$. (I) Quantification of podocyte markers WT-1-positive cells per glomerular section. The number of WT-1-stained nuclei was obtained from 20 glomeruli per kidney section from five mice per group. (m) Absolute count of the numbers of glomerular cells positive for both TUNEL and WT-1 (white arrows), data were expressed as the means of apoptotic podocytes from 20 randomly selected glomeruli. All above data were from at least three independent experiments. ${ }^{*} P<0.05$ versus controls 
a
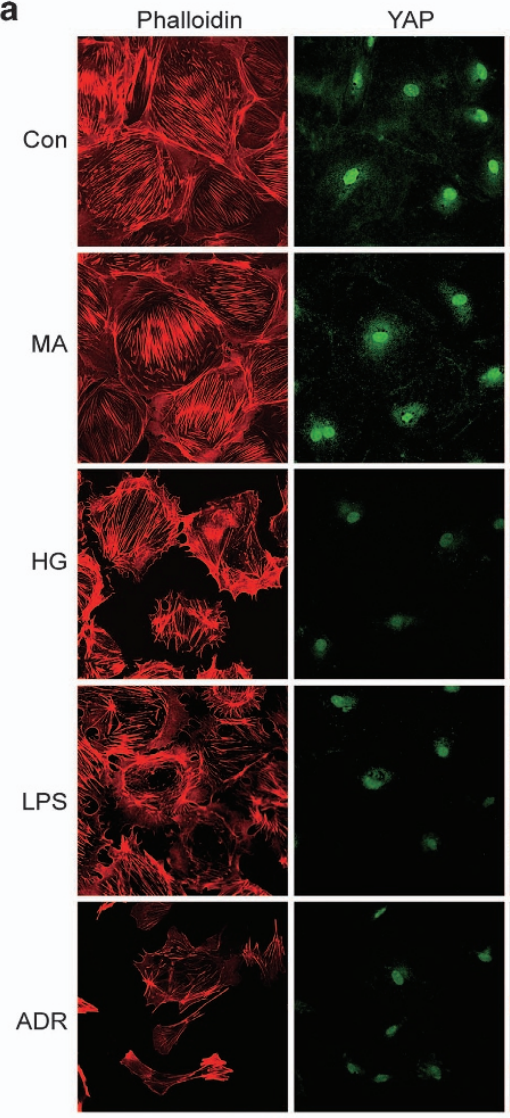
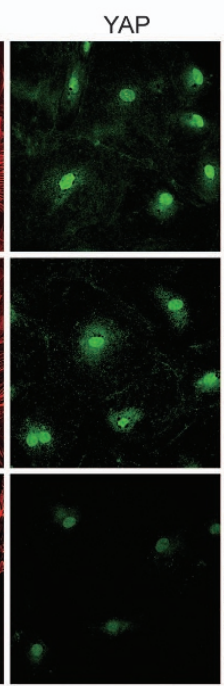
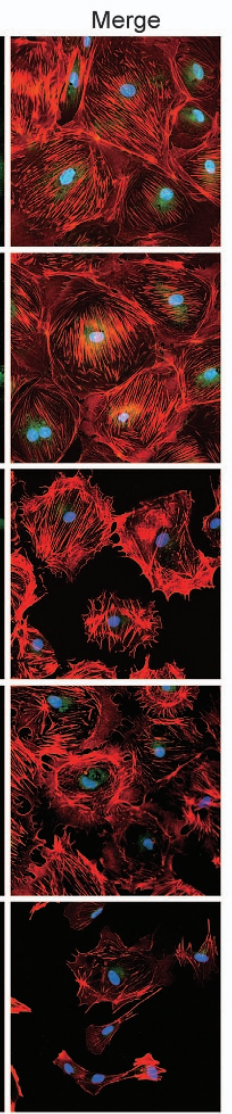

b

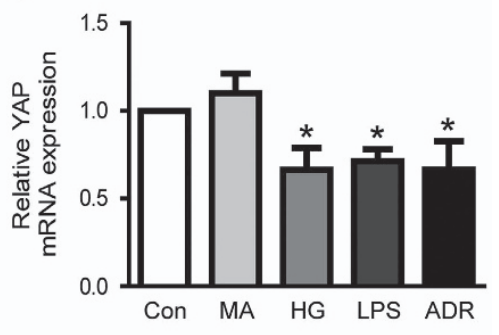

C
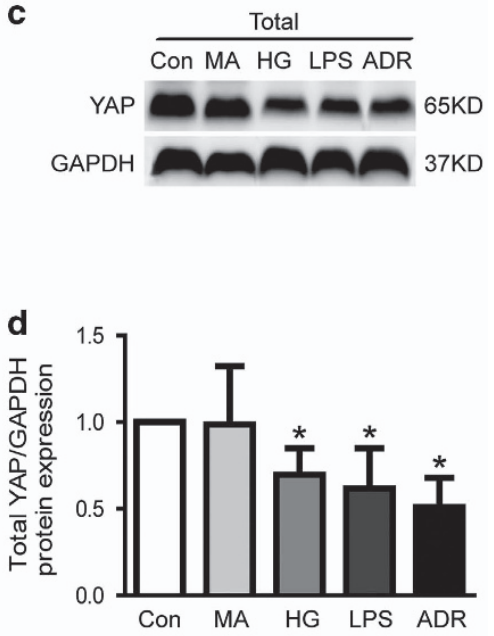

LS


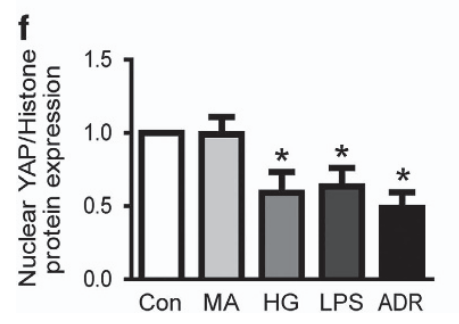

h
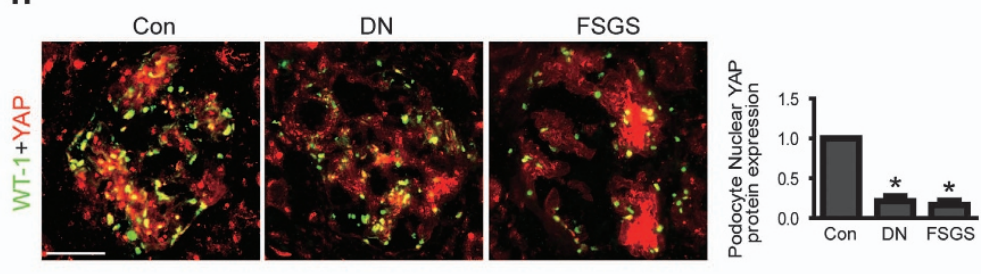

Figure 4 YAP and stress fiber were decreased in injured podocytes. (a) Typical confocal images of culture podocytes showing expression of YAP (green), Phalloidin-stained stress fiber (red) and DAPI-stained nuclei (blue). Compared to Con, nuclear and cytoplasmic YAP and stress fiber were obviously reduced in HG, LPS or ADR-injured podocytes. (b) The mRNA expression of YAP was decreased in HG, LPS or ADR-injured podocytes. (c-f) Total and nuclear protein expression of YAP was decreased in HG, LPS or ADRinjured podocytes. (g) Representative micrographs of dual-color fluorescence staining of kidney glomeruli for WT-1 (green) and YAP (red) from control, LPS and ADR mice. Magnification $\times 400$, scale bar $=25 \mu \mathrm{m}$. (h) Representative micrographs of dual-color fluorescence staining of kidney glomeruli for WT-1 (green) and YAP (red) from control, DN and FSGS patients. Magnification $\times 400$, scale bar $=25 \mu \mathrm{m}$. All above data were from at least three independent experiments. ${ }^{*} P<0.05$ versus controls

(Figures 5f-i). These results indicated that YAP mediated podocyte apoptosis was induced by HG, LPS or ADR.

Loss of Cdc42/Nwasp-decreased YAP expression in podocytes. To further explore the underlying mechanism of Cdc42-induced podocyte apoptosis, we also investigated the expression of its downstream effector Nwasp and YAP in Cdc42 knockdown podocytes. As shown in Figures $6 \mathrm{~g}-\mathrm{i}$, the results of real-time-qPCR and western blot analysis indicated that the mRNA and protein of Nwasp were markedly decreased in Cdc42 knockdown podocytes. Results from both immunofluorescence and western blotting demonstrated 
a

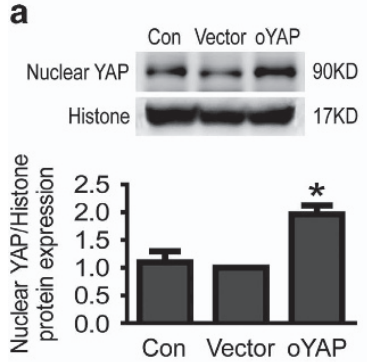

d

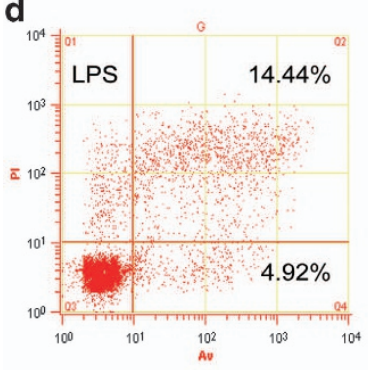

g

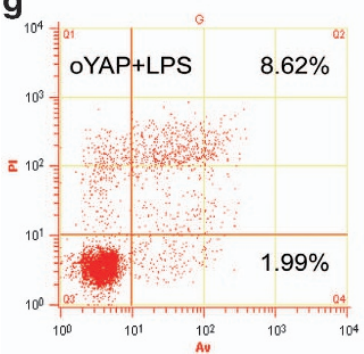

b

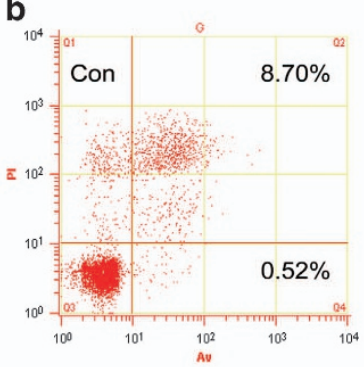

e

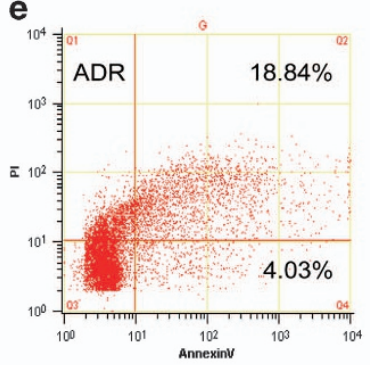

h

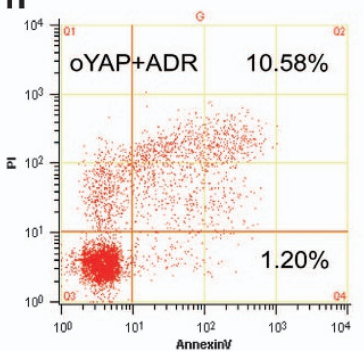

C



f

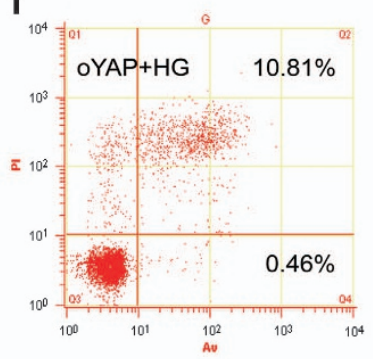

i

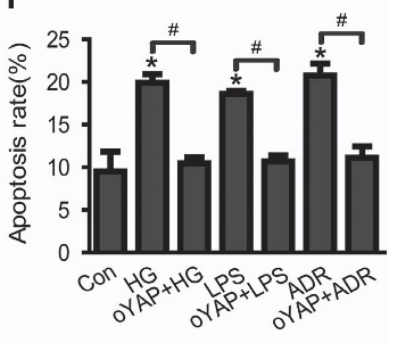

Figure 5 Overexpression of active YAP alleviates podocyte apoptosis induced by HG, LPS or ADR. (a) Nuclear YAP protein expression was obviously increased in overexpression of active YAP in podocytes. (b-e) Podocytes were stained with Annexin V/PI for flow cytometry analysis. Cell apoptosis rate was significantly increased in HG, LPS or ADR-treated podocytes. (f-i) Apoptosis induced by HG, LPS or ADR was significantly alleviated in overexpression of active YAP in podocytes. ${ }^{*} P<0.05$ versus Con, ${ }^{\sharp} P<0.05$

that both nuclear and cytoplasmic YAP protein expression was obviously decreased in Cdc42 or Nwasp knockdown podocytes (Figures 6a, c-f). Similar results from real-timeqPCR to analysis the mRNA expression of YAP were also obtained in Cdc42 or Nwasp knockdown podocytes (Figure 6b). Furthermore, specifically inhibited Cdc42 expression with ML-141 in mice also decreased nuclear YAP protein expression (Figure 6j). Thus, these data strongly indicated that $\mathrm{Cdc} 42 / \mathrm{Nwasp}$ could positively regulate YAP expression.

Stress fiber/YAP-mediated Cdc42/Nwasp deficiencyinduced podocyte apoptosis. To further explore the underlying mechanism of Cdc42/Nwasp regulating YAP, we firstly investigated the expression of Phalloidin-stained stress fiber, which had been regarded as a regulator of YAP, ${ }^{20,21}$ in Cdc42 or Nwasp knockdown podocytes. As shown in Figure 6a, Phalloidin-stained stress fiber was decreased in vitro Cdc42 or Nwasp knockdown podocytes accompanied with increased podocyte apoptosis (Figures $3 a-f)$. This was consistent with above results that stress fiber was also decreased (Figure 4a) as podocyte apoptosis was increased (Figures 1g-I) in HG, LPS or ADR-injured podocytes.
Then, we explored the role of stress fiber in podocytes apoptosis. As shown in Figures $7 \mathrm{a}-\mathrm{j}$, inhibited stress fiber formation with the specific inhibitor LatA resulted in increased podocyte apoptosis accompanied with decreased YAP mRNA and protein expression, but not changed the protein expression of Cdc42 and Nwasp. To further confirmed Cdc42/Nwasp/stress fiber-mediated podocyte apoptosis through YAP, we also investigated the apoptotic effect of overexpression of active YAP in Cdc42 or Nwasp knockdown podocytes or LatA-treated podocytes. As shown in Figures $8 \mathrm{a}-\mathrm{j}$, podocyte apoptosis induced by $\mathrm{Cdc} 42$ or Nwasp knockdown or LatA treatment was significantly abolished by overexpressing active YAP. These data mentioned above indicated that the stress fiber/YAP probably mediated Cdc42/Nwasp deficiency, induced podocyte apoptosis.

\section{Discussion}

Podocytes are very special cells with complex actin cytoskeletal architecture, which play a pivotal role in maintaining normal glomerular function. ${ }^{25}$ Loss of podocyte caused by apoptosis is commonly happened in the pathogenesis 
of many kidney diseases, such as diabetic nephropathy, ${ }^{2,3}$ focal segmental glomerular sclerosis, ${ }^{4,26}$ et al. However, the mechanism-mediating podocyte apoptosis remains incompletely understood. Therefore, exploring the mechanisms that cause podocyte apoptosis in these kidney diseases is imperative. a



b

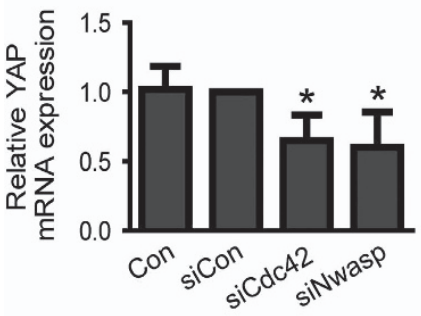

e

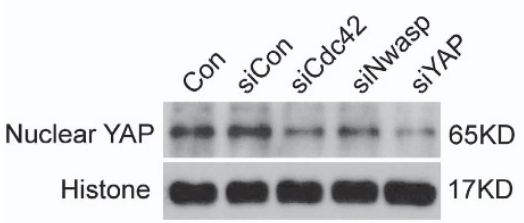

h
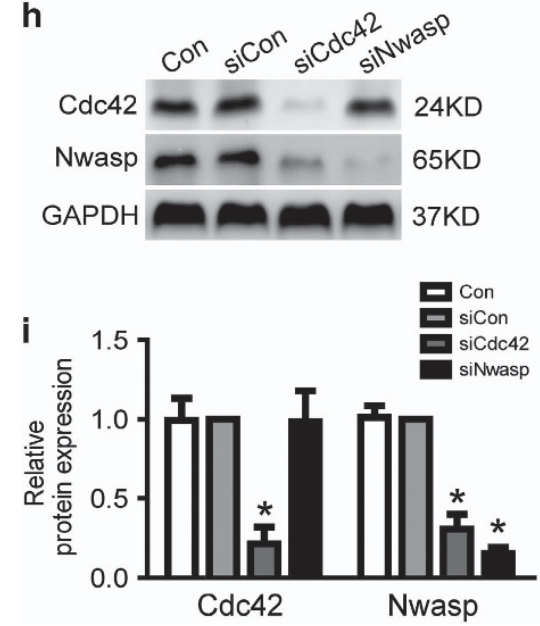

c

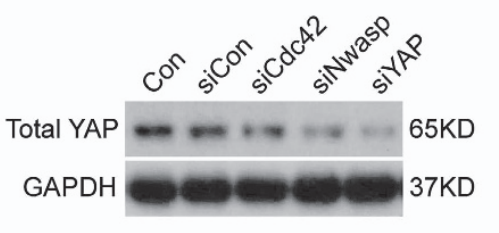

f
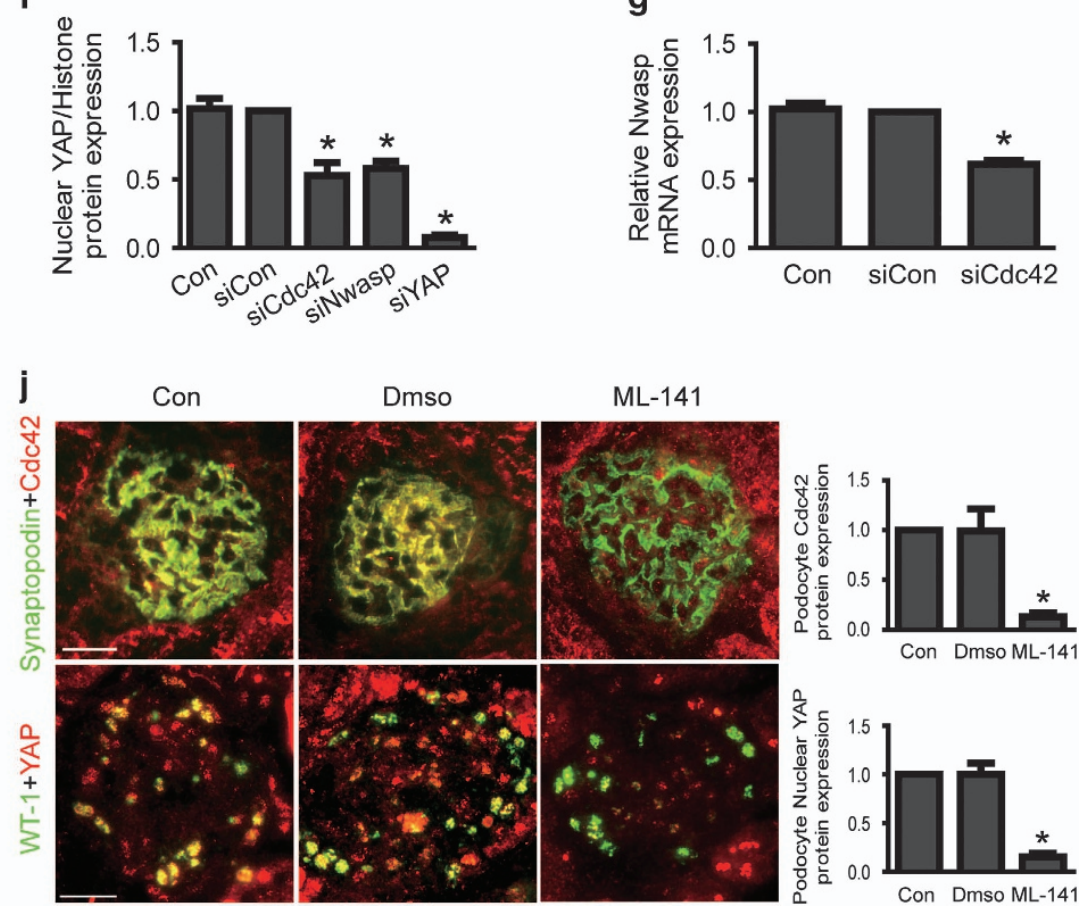
In this study, we found that Cdc42 expression and its activity were significantly decreased in injured podocytes induced by HG or LPS or ADR. Decreased Cdc42 expression is also observed in podocytes of patients with diabetic DN or FSGS. Moreover, we found that podocyte apoptosis was increased in
Cdc42 deficiency podocytes in vivo and in vitro. We also further demonstrated that the loss of Cdc42 or its downstream effector Nwasp-induced podocyte apoptosis was probably mediated by decreased stress fibers/YAP expression, which the latter had been regarded as an anti-apoptosis $\mathbf{a}_{10^{4}}$
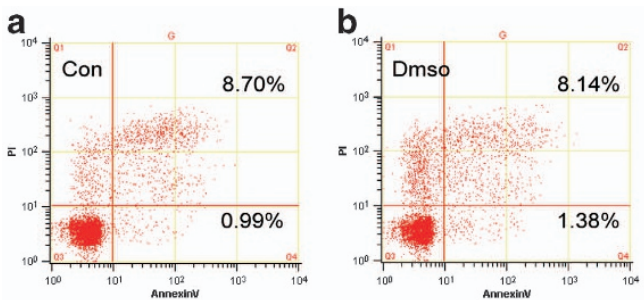

e

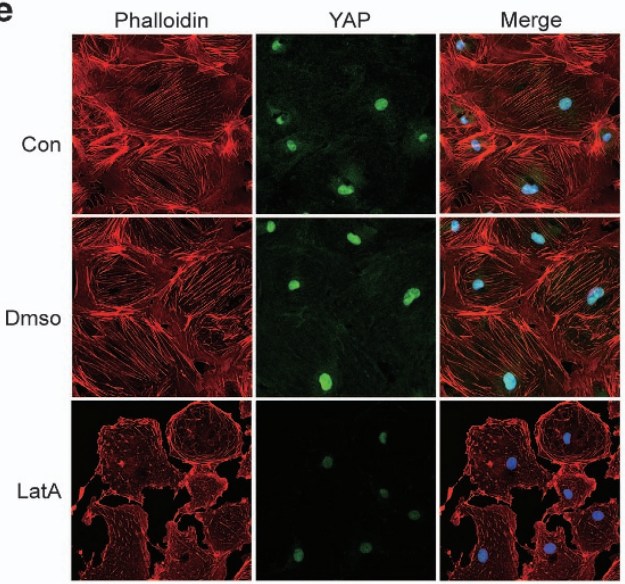

C

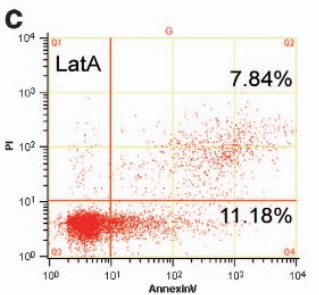

d

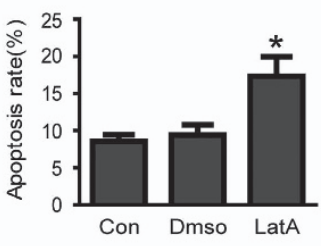

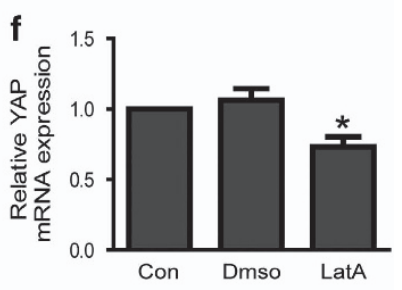

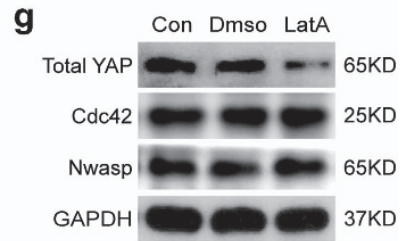

h
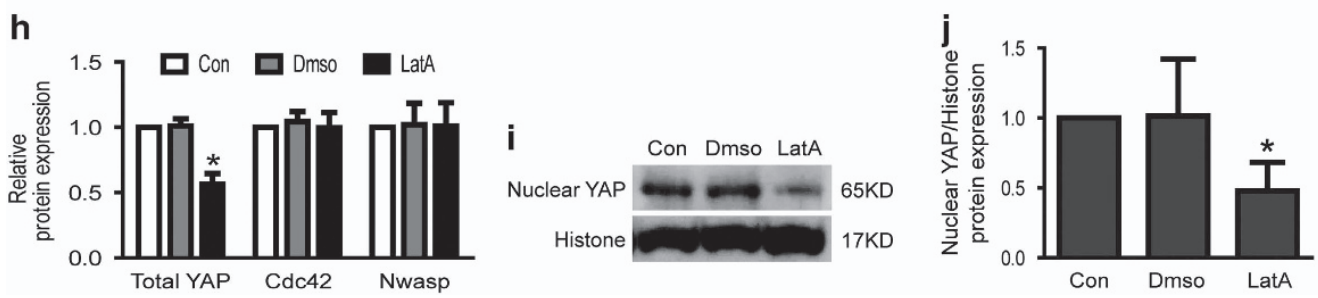

Figure 7 Inhibited formation of stress fiber induced podocyte apoptosis and decreased YAP expression. (a-d) Podocytes were stained with Annexin V/PI for flow cytometry analysis. Cell apoptosis rate was significantly increased in LatA-(F-actin inhibitor, inhibited stress fiber formation) treated podocytes. (e) Typical confocal images of culture podocytes showing expression of YAP (green), Phalloidin-stained stress fiber (red) and DAPI-stained nuclei (blue). Compared to Con, nuclear and cytoplasmic YAP and stress fiber were obviously reduced in LatA-treated podocytes. (f) The mRNA expression of YAP was decreased in LatA-treated podocytes. (g-j) Total and nuclear protein expression of YAP was decreased in LatA-treated podocytes, but the protein expression of Cdc42 and Nwasp were not changed. All above data were from at least three independent experiments. ${ }^{*} P<0.05$ versus controls

Figure 6 Loss of Cdc42/Nwasp decreased stress fiber formation and YAP expression. (a) Typical confocal images of culture podocytes showing expression of YAP (green), Phalloidin-stained stress fiber (red) and DAPI-stained nuclei (blue). Compared to siCon, nuclear and cytoplasmic YAP and stress fiber were obviously reduced in Cdc42 siRNA or Nwasp siRNA-treated podocytes. (b) The mRNA expression of YAP was decreased in Cdc42 siRNA and Nwasp siRNA-treated podocytes. (c-f) The total and nuclear protein expression of YAP was decreased in Cdc42 siRNA or Nwasp siRNA-treated podocytes. (g) The mRNA expression of Nwasp was decreased in Cdc42 siRNA-treated podocytes. (h-i) Cdc42 and Nwasp protein expression were reduced to about 21.8\%, 15.4\% in Cdc42 siRNA and Nwasp siRNA-treated podocytes, respectively. Nwasp protein expression was obviously decreased in Cdc42 knockdown podocytes comparing to siCon, as Cdc42 protein expression was not changed in Nwasp knockdown podocytes. (j) On top: typical images of dual-color fluorescence staining of kidney glomeruli for synaptopodin (green) and Cdc42 (red) from control, Dmso and ML-141 (Cdc42-specific inhibitor) mice. Cdc42 was hardly seen in podocytes from ML-141 mice. Below: representative micrographs of dual-color fluorescence staining of kidney glomeruli for WT-1 (green) and YAP (red) from control, Dmso and ML-141 mice. Nuclear YAP was decreased in podocytes from ML-141 mice comparing to Con. Magnification $\times 400, s c a l e$ bar $=25 \mu \mathrm{m}$. All above data were from at least three independent experiments. ${ }^{*} P<0.05$ versus controls 

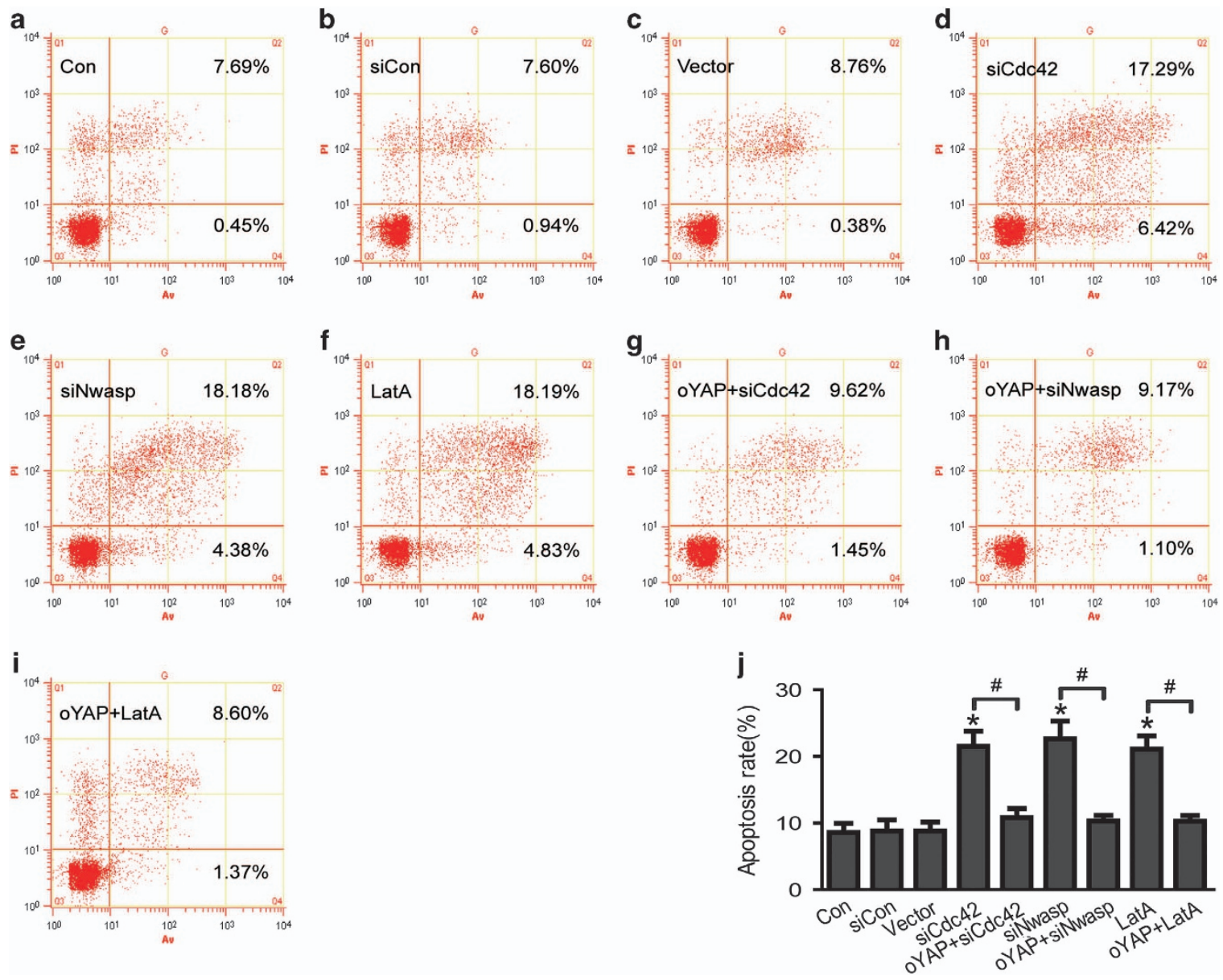

Figure 8 Overexpression of active YAP decreased podocyte apoptosis induced by Cdc42 or Nwasp knockdown podocytes or LatA-treated podocytes. Podocytes were stained with Annexin V/PI for flow cytometry analysis. Con, siCon, vector, oYAP, siCdc42, siNwasp, siYAP were short for blank control, control siRNA, overexpression vector, overexpressed-active YAP, Cdc42 siRNA, Nwasp siRNA, YAP siRNA, respectively. (a-f) Cell apoptosis rate was significantly increased in Cdc42 or Nwasp knockdown podocytes or LatA-(F-actin inhibitor, inhibited stress fiber formation) treated podocytes. (g-j) Podocyte apoptosis induced by Cdc42 or Nwasp knockdown or LatA treatment was significantly inhibited by overexpressing active YAP. All above data were from at least three independent experiments. ${ }^{*} P<0.05$ versus controls, ${ }^{\#} P<0.05$

protein in podocyte. ${ }^{18,19}$ Collectively, these data indicated that the Cdc42/Nwasp/stress fibers/YAP signal pathway may potentially play an important role in regulating podocyte apoptosis.

The function of podocytes is regulated by small GTPases belonging to the Rho GTPase family. ${ }^{7}$ These small GTPases act as molecular switches to control the activation of multiple downstream effectors. ${ }^{27-30}$ Among their pleiotropic actions, Rho-dependent signaling cascades modulate cellular morphology and actin polymerization, adhesion, cell migration, proliferation and apoptosis. ${ }^{20,27-31}$ Cdc42 is one of the Rho-GTPases that have been well studied, and has been regarded as a main regulator of the function of cytoskeletal architecture. $^{11,12}$ It mediates multiple signaling pathways, including tyrosine kinase receptors, heterotrimeric G-proteincoupled receptors, cytokine receptors, integrins, and physical and chemical stress. ${ }^{32}$ Recent studies demonstrated Cdc42 has a crucial role in podocyte cell maintenance. ${ }^{33}$ Mice only lacking Cdc42 showed early embryonic lethality and defective actin cytoskeleton. ${ }^{11}$ Podocyte-specific deletion of Cdc42, but not Rac1, resulted in many overt changes to podocyte morphology and overall glomerular function. ${ }^{12}$ Here, in our study, we firstly focused on the response of Cdc42 to podocyte injury. We investigated the expression of Cdc42 in human nephropathy with proteinuria. Interestingly, Cdc42 protein expression and activity were significantly decreased or inhibited in injured podocyte induced by HG, LPS and ADR. Similarly, decreased Cdc42 expression was also detected in glomerular podocyte of FSGS and DN patients.

Based on pleiotropic actions of $\mathrm{Cdc} 42,{ }^{20,31}$ we speculated that the reduction of $\mathrm{Cdc} 42$ in the podocyte as a response to injury might be related to podocyte apoptosis. Therefore, we next sought to investigate whether the loss of Cdc42 induces podocyte apoptosis. Indeed, we observed increased apoptotic podocytes by both TUNEL assay and Annexin V, and propidium iodide staining after knockdown of endogenic Cdc42 gene in vitro and inhibition of Cdc42 activity in vivo. Meanwhile, the number of podocytes was significantly decreased after inhibition of Cdc42 activity with ML-141 in vivo. In addition, apoptosis-related genes $\mathrm{Bax}$ and $\mathrm{Bcl} 2$ were also assessed by real-time PCR and immunoblot, and significant aberrant expressions were found. These data 
demonstrated that insufficient Cdc42 could cause podocyte apoptosis, which is another main reason that will cause proteinuria. This indicates that the podocyte apoptosis induced by $\mathrm{Cdc} 42$ deficiency is probably a new mechanism of proteinuria.

To further explore the mechanisms of Cdc42 deficiencymediated podocyte apoptosis, we investigated the expression of its downstream effector Nwasp and transcriptional co-activator YAP. $^{34}$ Numerous studies have demonstrated that the Rho GTPase Cdc42 controls actin dynamics by regulating actin polymerization stability via its downstream target Nwasp in vitro. ${ }^{30,35}$ In present study, we found that Nwasp protein expression was significantly decreased in Cdc42 knockdown podocytes, however, Cdc42 protein expression was not changed in Nwasp knockdown podocytes, it was consistent with previous results that the Nwasp protein was downstream target of Rho GTPase Cdc42. In addition, we also demonstrated that apoptosis was increased in Nwasp knockdown podocytes. The apoptosis-related marker Bax and $\mathrm{Bcl} 2$ were also found to be activated and inhibited respectively accompanied with decreased Cdc42 and Nwasp. These further suggest that Cdc42/Nwasp is implicated in podocytes apoptosis.

YAP is a transcriptional co-activator that regulates cell proliferation and apoptosis downstream of the Hippo kinase pathway. Dephosphorylated YAP localizes in the nucleus and functions as a transcriptional co-activator to induce target gene expression in normal condition. ${ }^{15}$ It has a well-described role in controlling the expression of genes that promote cell proliferation and inhibit apoptosis. ${ }^{13,14,18}$ In the current study, we found that the total and nuclear YAP protein expression were significantly decreased in HG, or LPS or ADR-mediated podocyte injury in vivo and in vitro. Similar results were also obtained in patients with DN and FSGS. Evidence had showed that YAP could also serve as a physiologic inhibitor of podocyte apoptosis. ${ }^{18}$ This was consistent with our results that apoptosis was increased in YAP knockdown podocytes. In addition, the apoptosis-related marker $\mathrm{Bax}$ and $\mathrm{Bcl} 2$ were also found to be aberrant in YAP knockdown podocytes.

Previous studies had identified YAP/TAZ as sensors and mediators of mechanical cues instructed by the cellular microenvironment. ${ }^{21,36}$ Cdc42/Nwasp is also known to regulate the dynamic organization of the cytoskeleton. ${ }^{30,34}$ Therefore, it's possible that Cdc42/Nwasp could positively regulate YAP in podocytes. As anticipated, our data further demonstrated that both nuclear and cytoplasmic protein expression of YAP were decreased in Cdc42 or Nwasp knockdown podocytes. Similar results were also obtained in glomerular podocyte of mice treated with specific Cdc42 inhibitor ML-141. ${ }^{37}$ It is consistent with the previous studies that Rho-GTPases could positively regulate YAP transcriptional activity ${ }^{21}$ and protein expression. ${ }^{38}$ Although previous study also showed that the inhibition of Cdc42 caused YAP inhibition, mainly by YAP sequestration in the cytoplasm in mouse embryonic fibroblasts, ${ }^{20}$ it's possible that Cdc42 could positively regulate YAP expression in podocytes. However, the mechanism remains to study further. Whatever, these data further suggests that Cdc42/Nwasp/YAP is probably implicated in podocyte apoptosis.
To further explore the underlying mechanism of Cdc42/ Nwasp regulate YAP, we focus on a cytoskeleton molecule Phalloidin-stained stress fiber, which had been regarded as a regulator of YAP. ${ }^{21}$ Previous studies had showed that Cdc42 could work through actin cytoskeleton to promote stress fibers formation. Similarly, in our study we found that podocyte deficiency in Cdc42 or Nwasp could significantly lead to loss of stress fiber, decreased YAP expression and increased podocyte apoptosis. This was consistent with results that stress fiber was also decreased in HG, LPS or ADR-treated podocytes with increased podocyte apoptosis. Inhibited stress fiber with specific inhibitor Lat $A^{21}$ resulted in increased podocyte apoptosis accompanied with decreased nuclear and cytoplasmic YAP expression, but not changed the protein expression of Cdc42 and Nwasp. These results indicated that YAP was the downstream molecule of stress fiber, but not Cdc42 and Nwasp.

To further confirmed Cdc42/Nwasp/stress fiber-mediated podocyte apoptosis through YAP, we also investigated the apoptotic effect of overexpression of active YAP in Cdc42/ Nwasp/stress fiber deficiency podocytes. We found that the podocyte apoptosis induced by Cdc42 or Nwasp knockdown or LatA treatment was significantly alleviated by overexpressing active YAP. These results indicated that stress fiber/YAP probably mediated Cdc42/Nwasp deficiency-induced podocyte apoptosis. Therefore, Cdc42/Nwasp/stress fibers/YAP may be an important signal pathway to regulate podocyte apoptosis. Insufficient Cdc42/Nwasp/stress fibers/YAP pathway activity in podocyte may be one common reason that causes proteinuria in kidney diseases including FSGS and DN.

Taken together, the present study demonstrated that the expression of Cdc42 and its activity were significantly decreased as cell apoptosis was increased in injured podocyte in vitro and in vivo. Decreased Cdc42 expression by SiRNA and selective inhibitor ML-141 in vitro and in vivo caused podocyte apoptosis and proteinuria, accompanied with increased pro-apoptotic Bax and decreased antiapoptotic $\mathrm{Bcl}-2$ gene and protein expression. Our results further demonstrated that insufficient Cdc42 or Nwasp, its downstream effector, could decrease the mRNA and protein expression of YAP, which had been regarded as an antiapoptosis protein in podocyte. Moreover, our data indicated that loss of stress fibers caused by Cdc42/Nwasp deficiency also decreased YAP mRNA and protein expression, and induced podocyte apoptosis. Podocyte apoptosis induced by Cdc42/Nwasp/stress fibers deficiency was significantly abolished by overexpressed-active YAP. Therefore, the Cdc42/ Nwasp/stress fibers/YAP signal pathway may potentially play an important role in regulating podocyte apoptosis. Maintaining necessary Cdc42 would be one potent way to prevent proteinuria kidney diseases.

\section{Materials and Methods}

Cell culture and treatment. The conditionally immortalized mouse podocyte cell line was a kind gift from Dr Jochen Reiser (Rush University Medical Center, Chicago, IL, USA), and cultured as described previously. ${ }^{39}$ Cells were cultured at $33^{\circ} \mathrm{C}$ in RPMI-1640 medium (Gibco BRL, Gaithersburg, MD, USA) supplemented with $10 \%$ fetal bovine serum (FBS, Gibco BRL, Gaithersburg, MD, USA) and recombinant IFN- $\gamma$ (growth permissive conditions; CYT-358, ProSpec, Tany 
Technogene Ltd, Ness Ziona, Israel). To induce differentiation, podocytes were reseeded and cultured at $37^{\circ} \mathrm{C}$ in $100 \mathrm{~cm}^{2}$ culture dish coated with $12 \mathrm{mg} / \mathrm{ml}$ type-l collagen (BD Bioscience, Bedford, MA, USA) and in Ddulbecco's modified eagle medium (with $5.3 \mathrm{mM}$ glucose, Invitrogen, Carlsbad, CA, USA) supplemented with $5 \%$ FBS, deprived of IFN- $\gamma$ (growth restrictive conditions) for 10-13 days. After differentiation, podocytes was confirmed by the identification of synaptopodin, a podocyte differentiation marker, $10^{6}$ cells were synchronized into quiescence by growing cells in serum-free RPMl-1640 medium (Gibco BRL) for $24 \mathrm{~h}$, and then treated with MA, HG (add to $30 \mathrm{mM}$ glucose), LPS $(100 \mu \mathrm{g} / \mathrm{ml})$, ADR $(0.125 \mu \mathrm{g} / \mathrm{ml})$ or siRNA $(50 \mathrm{nM})$ for $48 \mathrm{~h}$. Each reaction was repeated in triplicates.

Transfection of siRNAs and plasmids. The siRNAs against Cdc42, Nwasp, YAP, and control were designed and synthesized by RiboBio Co. Ltd (Guangzhou, China). They were transfect into podocytes using Lipofectamine 2000 reagent (Invitrogen) following the manufacturer's protocol. The sequences of siRNAs used in this study were as follows: siCdc42 5'GCAAGAGGAUUAUGACAGA dTdT-3', siNwasp 5'GGUGUCGCUUGUCUGGUUA dTdT-3', siYAP 5'-CGAGAUGAGAGCACA GACA dTdT-3'. Lentiviral packaging wild-type YAP or YAP S112A mutation plasmids (CS-Mm06093-Lv128, GeneCopoeia, Guangzhou, China) were generated in HEK293T cells that were expanded and transfected using the Lenti-Pac HIV Expression Packing Kit (HPK-LvTR-20, GeneCopoeia) according to the manufacturer's protocols. Podocytes were then infected in 6-well plate (Trueline, Salt Lake, UT, USA) with the lentivirus particles. Forty eight hours after transfection, the cells under different conditions were collected. Each transfection was repeated in triplicate.

Real-time quantitative-PCR. As described previously, ${ }^{2}$ RNA samples were prepared using Trizol RNA isolation system (Invitrogen) and reverse transcribed into cDNAs using the PrimeScriptTM RT regent Kit following the instructions provided by the manufacturer (Takara Bio Inc, Otsu, Shiga, Japan), and then cDNAs were used for real-time PCR analysis by a Plantinum SYBR Green SuperMix-UDG kit (Takara Bio Inc). The primers used are listed as follows: Cdc42, forward 5'-ATTATGACAGACTACGACCGCT-3', reverse 5'-AGTGGTGAGTTATCTCA GGCA-3'; Nwasp, forward 5'-ATGCTCCAAATGGTCCCAATC-3', reverse 5'-CTTGG TGTTCCAATATCTGCCT-3' YAP, forward 5'-TGAGATCCCTGATGATGTACCAC-3', reverse 5'-TGTTGTTGTCTGATCGTTGTGAT-3'; Bax, forward 5'-CTGGACCATAGG TCGGAGTG-3', reverse 5'-AATTCGCCGGAGACACTCG-3'; Bcl-2, forward 5'-GTC GCTACCGTCGTGACTTC-3', reverse 5'CAGACATGCACCTACCCAGC-3'; GAPDH, forward 5'-AGGTCGGTGTGAACGG ATTTG-3', reverse 5'-TGTAGACCATGTAGTTG AGGTCA-3'.

Western blotting. Whole and nuclear proteins were prepared as described previously. ${ }^{2}$ An aliquot of cell lysates containing $30 \mu \mathrm{g}$ of protein was separated on $10 \%$ sodium dodecyl sulfate-polyacrylamide gels, and then transferred to polyvinylidene fluoride membranes (Millipore, Billerica, MA, USA). After blocked by $5 \%$ non-fat dry milk for $1 \mathrm{~h}$ at room temperature, membranes were incubated overnight at $4{ }^{\circ} \mathrm{C}$ with the following primary antibodies: rabbit anti-YAP (Cell Signaling Technology, Danvers, MA, USA, 1:1000), rabbit anti-Histone (Cell Signaling Technology, 1:3000), rabbit anti-Cdc42 (Santa Cruz, Dallas, TX, USA, 1:500), rabbit anti-Nwasp (Santa Cruz, 1:500), rabbit anti-Bax (Santa Cruz, 1:500), rabbit anti-Bcl-2 (Cell Signaling Technology, 1:1000), rabbit anti-GAPDH (Bioworld Technology, Nanjing, China, 1:10 000). The membranes were then incubated with anti-rabbit IgG (Jackson Immuno Research, West Grove, PA, USA, 1: 4000) at room temperature for $1 \mathrm{~h}$. Finally, membranes were treated with $\mathrm{ECL}$ reagents (Advansta, Menio Park, CA, USA), followed by exposing to X-ray film (Kodak, Rochester, NY, USA). The bands of the resulting autoradiographs were quantified densitometrically using Bandscan software. Protein expression was quantified as the ratio of specific band to Histone (nuclear fractions) or GAPDH.

Annexin V and propidium iodide (PI) staining assay. Apoptotic cells in different groups were determined using an Annexin V/PI apoptosis detection kit according to manufacturer's protocol (Nanjing KeyGEN Biotech, Nanjin, China). Briefly, podocytes were resuspended with binding buffer followed by incubation with $5 \mathrm{ml}$ of Annexin V (conjugated with FITC) and $5 \mathrm{ml}$ of Pl in the dark for $10 \mathrm{~min}$. Cell fluorescence was then analyzed using a Cell Lab QuantaTM SC Flow cytometer (Beckman Colter, Inc, USA). Cells positive for Annexin V-FITC were considered as apoptosis.

Cdc42 GTPase activation assay. Active Cdc42 was measured by G-LISA Cdc42 Activation Assay Biochem kit (colorimetric assay, Cytoskeleton, Denver, CO, USA) following the manufacturer's instruction. And the signal was measured at
$490 \mathrm{~nm}$ with a microplate reader (MRX, Dynatech Laboratories, Chantilly, VA, USA). Total Cdc42 protein expression of each group was measured by WB, as described above. Cell lysate from each group containing the same amount of protein was measured in both Cdc42 GTPase activation assay and WB. Results were expressed as fold activity of stimulated in relation to non-stimulated controls normalized to total Cdc42 protein content.

Patient studies. Renal biopsies tissues from patients with DN, FSGS and renal cell carcinoma (adjacent normal tissues) were respectively obtained after patients gave written consent. The study of patients was conducted in accordance with the Second Helsinki Declaration and approved by the Ethics Committee for Human Research of Guangdong General Hospital.

Animal studies. Animal care and experiments were performed in accordance with the ARRIVE guidelines, ${ }^{40}$ and were approved by the Ethics Committee for animal research of Guangdong General Hospital. Six male C57BL/KsJ db/db mice and 5 age-matched wild-type (BKS) mice were purchased from Model Animal Research Center of Nanjing University. Thirty-six male BALB/c mice aged 8 weeks were purchased from Center of Laboratory Animal Science of Guangdong. Mice were anaesthetized (ketamine, $70 \mathrm{mg} / \mathrm{kg}$, intraperitoneally (i.p.)) before killed and kidney tissue were collected. Mice urinary albumin and creatinine were measured using mouse albumin-specific ELISA (Bethyl Laboratories Inc, Montgomery, TX, USA) and creatinine kits (Cayman Chemical, Ann Arbor, MI, USA) respectively, according to the manufacturer's instructions. Proteinuria was expressed as the ratio of albumin to creatinine.

Diabetic mice model. The C57BL/KsJ db/db mice were obese and known to develop type 2 diabetes, followed by diabetic kidney disease. For urine collection, individual mice were caged once every 2 weeks in a metabolic cage for $24 \mathrm{~h}$ from week 12-week 20.

Murine models of LPS-induced proteinuria and podocytopathy. Five male BALB/c mice were randomized to each of the following treatments: LPSinduced proteinuric mice were i.p. injected with $200 \mu \mathrm{g}$ LPS $(1 \mathrm{mg} / \mathrm{ml}$ in sterile LPSfree saline, serotype: E. coli 0111:B4, Sigma, St Louis, MO, USA) in a total volume of $200 \mu$ l; control mice were i.p. injected with equal volumes of sterile LPS-free saline. ${ }^{41}$ Mice were followed for $24 \mathrm{~h}$ before they were killed and the kidneys resected for further investigation. Urine was collected before and $24 \mathrm{~h}$ after LPS injection.

Murine models of ADR-induced proteinuria and podocytopathy. Five male BALB/c mice were injected once with ADR $(2 \mathrm{mg} / \mathrm{ml}$ in sterile ADR-free saline, doxorubicin hydrochloride, Sigma) at a dose of $12 \mathrm{mg} / \mathrm{kg}$ body weight via the tail vein on day 0 . Five control BALB/c mice were injected with an equal volume of saline only. ${ }^{42}$ Urine of all mice was collected on day $0,4,7,14$.

Murine models of Cdc42 inhibition. Five male BALB/c mice were i.p. injected with $100 \mu \mathrm{l} \mathrm{ML-141}$ (selective inhibitor of Cdc42, $1 \mathrm{mg} / \mathrm{ml}$ in $30 \%$ dimethylsulfoxide-saline, Tocris Bioscience, Bristol, UK) daily for 3 weeks. ${ }^{37,43,44}$ Five blank control mice and five DMSO control mice were i.p. injected with $100 \mu \mathrm{l}$ saline and $30 \%$ dimethylsulfoxide-saline respectively everyday for 3 weeks. Urine of all mice was collected weekly before killed.

Podocyte number counting. The number of podocytes per glomerulus was determined based on methods described previously. ${ }^{45,46}$ Paraffin embedded tissue sections were deparaffinized and hydrated with water, then were placed in preheated $10 \mathrm{mM}$ Tris-buffered saline with $1.0 \mathrm{mM}$ EDTA (pH 9.0) and heated for $20 \mathrm{~min}$ at $100{ }^{\circ} \mathrm{C}$ in a water bath. After cooling to room temperature (RT), slides were rinsed in deionized water and then immersed in $3 \% \mathrm{H}_{2} \mathrm{O}_{2}$ to quench endogenous peroxidase activity. After being washed, slides were incubated with rabbit polyclonal antibody Wilm's tumor antigen 1 (WT-1; sc-192, Santa Cruz) for 60 min at RT, then washed and immunoperoxidase staining was performed using the Envision Plus system for rabbit primary antibodies (Dako Cytomation, Carpinteria, CA, USA) according to the manufacturer's instructions. The slides were counterstained with haematoxylin and permanently mounted before examination by light microscopy. The number of podocytes per glomerulus is the average number of nuclei stained in 20 randomly selected glomeruli.

Immunofluorescent staining and TUNEL staining. Cultured podocytes planted on cover slides in six-well plates or frozen cryostat sections were fixed 
with $4 \%$ paraformaldehyde at RT for 20 min, permeabilized with $0.1 \%$ Triton X-100 for $5 \mathrm{~min}$, then blocked with $5 \%$ bovine serum albumin for $30 \mathrm{~min}$ at room temperature before further incubated overnight at $4{ }^{\circ} \mathrm{C}$ with the following primary antibodies: goat anti-synaptopodin (Santa Cruz, 1:100), goat anti-WT-1 (Santa Cruz, 1:100), rabbit anti-Cdc42 (Santa Cruz, 1:100), rabbit anti-YAP (Santa Cruz, 1:100). Then cultured podocytes or sections were washed three times with PBS for 5 min before the secondary antibodies (FITC-donkey anti-goat IgG 488, Protein Tech Group, Inc, Rosemont, IL, USA, 1:200; goat anti-rabbit Alexa Fluor 555, Cell Signaling Technology, 1:200; goat anti-rabbit Alexa Fluor 488, Cell Signaling Technology, 1:1000) were applied for $1 \mathrm{~h}$ at $\mathrm{RT}$ in the dark. Culture podocytes were stained with phalloidin (Cytoskeleton, 1:500) for $30 \mathrm{~min}$ and then with DAPI (Sigma, St. Louis, MO, USA) for 5 min at room temperature. Apoptotic cell death in kidney sections was detected by using the TUNEL kit (Roche Molecular Biochemicals, Mannheim, Germany) as described previously. ${ }^{41,47}$ Cells positive for both TUNEL and WT-1 are apoptotic podocytes. The quantifications of these data were expressed as the means of apoptotic podocytes from 20 randomly selected glomeruli. Photomicrographs were taken with laser confocal microscopy (LCSM, Zeiss KS 400, Postfach, Germany) and analyzed by Image-Pro Plus 6.0 (Media Cybernetics, Georgia Avenue, MD, USA) for quantification. All images were analyzed by two investigators blinded to the identity of the samples.

Statistical analysis. All values are expressed as mean \pm S.D. Statistical analysis was performed using the statistical package SPSS for Windows Ver. 19.0 (SPSS, Inc., Chicago, IL, USA). All experimental observations were repeated more than three times. Statistical analysis of the data from multiple groups was performed by analysis of variance followed by Student-Newman-Keuls tests. Data from two groups were compared by Student's $t$-test. $P$-values $<0.05$ were considered significant.

\section{Conflict of Interest}

The authors declare no conflict of interest.

Acknowledgements. This study was supported by National Clinical Key Specialty Construction Projects, National Natural Science Foundation of China (81470930, 81270784, 81500560, 81400738) and Natural Science Foundation of Guangdong Province, China (2014A030310107, 2015A030313531).

\section{Author contributions}

W Shi, ZS Huang and L Zhang designed this study. ZS Huang performed the experiments. L Zhang and ZS Huang wrote this paper. All the other authors helped to design and perform this study. All the authors read and approved the final manuscript.

1. Canaud G, Bienaime F, Viau A, Treins C, Baron W, Nguyen C et al. AKT2 is essential to maintain podocyte viability and function during chronic kidney disease. Nat Med 2013; 19 : $1288-1296$

2. Li R, Zhang L, Shi W, Zhang B, Liang X, Liu S et al. NFAT2 mediates high glucose-induced glomerular podocyte apoptosis through increased Bax expression. Exp Cell Res 2013; 319: 992-1000.

3. Susztak K, Raff AC, Schiffer M, Bottinger EP. Glucose-induced reactive oxygen species cause apoptosis of podocytes and podocyte depletion at the onset of diabetic nephropathy. Diabetes 2006; 55: 225-233.

4. Marshall CB, Krofft RD, Pippin JW, Shankland SJ. CDK inhibitor p21 is prosurvival in adriamycin-induced podocyte injury, in vitro and in vivo. Am J Physiol Renal Physiol 2010; 298: F1140-F1151.

5. Etienne-Manneville S, Hall A. Rho GTPases in cell biology. Nature 2002; 420: 629-635.

6. Jaffe AB, Hall A. Rho GTPases: biochemistry and biology. Annu Rev Cell Dev Biol 2005; 21: 247-269.

7. Mouawad F, Tsui H, Takano T. Role of Rho-GTPases and their regulatory proteins in glomerular podocyte function. Can J Physiol Pharmacol 2013; 91: 773-782.

8. Reiser J, Sever S. Podocyte biology and pathogenesis of kidney disease. Annu Rev Med 2013; 64: 357-366.

9. Wang L, Ellis MJ, Gomez JA, Eisner W, Fennell W, Howell DN et al. Mechanisms of the proteinuria induced by Rho GTPases. Kidney Int 2012; 81: 1075-1085.

10. Meyer-Schwesinger C, Dehde S, Sachs M, Mathey S, Arefi K, Gatzemeier S et al. Rhokinase inhibition prevents proteinuria in immune-complex-mediated antipodocyte nephritis. Am J Physiol Renal Physiol 2012; 303: F1015-F1025.

11. Scott RP, Hawley SP, Ruston J, Du J, Brakebusch C, Jones $N$ et al. Podocyte-specific loss of Cdc42 leads to congenital nephropathy. J Am Soc Nephrol 2012; 23: 1149-1154.
12. Blattner SM, Hodgin JB, Nishio M, Wylie SA, Saha J, Soofi AA et al. Divergent functions of the Rho GTPases Rac1 and Cdc42 in podocyte injury. Kidney Int 2013; 84: 920-930.

13. Pan D. The hippo signaling pathway in development and cancer. Dev Cell 2010; 19 : 491-505.

14. Huang J, Wu S, Barrera J, Matthews K, Pan D. The hippo signaling pathway coordinately regulates cell proliferation and apoptosis by inactivating Yorkie, the Drosophila homolog of YAP. Cell 2005; 122: 421-434.

15. Cao X, Pfaff SL, Gage FH. YAP regulates neural progenitor cell number via the TEA domain transcription factor. Genes Dev 2008; 22: 3320-3334.

16. Hao Y, Chun A, Cheung K, Rashidi B, Yang X. Tumor suppressor LATS1 is a negative regulator of oncogene YAP. J Biol Chem 2008; 283: 5496-5509.

17. Zhao B, Wei X, Li W, Udan RS, Yang Q, Kim J et al. Inactivation of YAP oncoprotein by the Hippo pathway is involved in cell contact inhibition and tissue growth control. Genes Dev 2007; 21: 2747-2761

18. Campbell KN, Wong JS, Gupta R, Asanuma K, Sudol M, He JC et al. Yes-associated protein (YAP) promotes cell survival by inhibiting proapoptotic dendrin signaling. J Biol Chem 2013; 288: 17057-17062.

19. Schwartzman M, Reginensi A, Wong JS, Basgen JM, Meliambro K, Nicholas SB et al. Podocyte-specific deletion of Yes-associated protein causes FSGS and progressive renal failure. J Am Soc Nephrol 2015; 27: 216-226.

20. Reginensi A, Scott RP, Gregorieff A, Bagherie-Lachidan M, Chung C, Lim DS et al. Yap- and Cdc42-dependent nephrogenesis and morphogenesis during mouse kidney development. PLOS Genet 2013; 9: e1003380.

21. Dupont S, Morsut L, Aragona M, Enzo E, Giulitti S, Cordenonsi M et al. Role of YAP/TAZ in mechanotransduction. Nature 2011; 474: 179-183.

22. Faul C, Donnelly M, Merscher-Gomez S, Chang YH, Franz S, Delfgaauw J et al. The actin cytoskeleton of kidney podocytes is a direct target of the antiproteinuric effect of cyclosporine A. Nat Med 2008; 14: 931-938.

23. Comper WD. Is the LPS-mediated proteinuria mouse model relevant to human kidney disease? Nat Med 2009; 15: 133 (author reply 133-134).

24. Lee VW, Harris DC. Adriamycin nephropathy: a model of focal segmental glomerulosclerosis. Nephrology (Carlton) 2011; 16: 30-38.

25. Fogo AB. The targeted podocyte. J Clin Invest 2011; 121: 2142-2145

26. Daehn I, Casalena G, Zhang T, Shi S, Fenninger F, Barasch N et al. Endothelial mitochondrial oxidative stress determines podocyte depletion in segmental glomerulosclerosis. J Clin Invest 2014; 124: 1608-1621.

27. Heasman SJ, Ridley AJ. Mammalian Rho GTPases: new insights into their functions from in vivo studies. Nat Rev Mol Cell Biol 2008; 9: 690-701.

28. Tapon N, Hall A. Rho, Rac and Cdc42 GTPases regulate the organization of the actin cytoskeleton. Curr Opin Cell Biol 1997; 9: 86-92.

29. Ma L, Rohatgi R, Kirschner MW. The Arp2/3 complex mediates actin polymerization induced by the small GTP-binding protein Cdc42. Proc Natl Acad Sci U S A 1998; 95: 15362-15367.

30. Rohatgi R, Ma L, Miki H, Lopez M, Kirchhausen T, Takenawa T et al. The interaction between N-WASP and the Arp2/3 complex links Cdc42-dependent signals to actin assembly. Cell 1999; 97: 221-231

31. Zugasti O, Rul W, Roux P, Peyssonnaux C, Eychene A, Franke TF et al. Raf-MEK-Erk cascade in anoikis is controlled by Rac1 and Cdc42 via Akt. Mol Cell Biol 2001; 21: 6706-6717.

32. Sinha S, Yang W. Cellular signaling for activation of Rho GTPase Cdc42. Cell Signal 2008 20: 1927-1934.

33. Yanagida-Asanuma E, Asanuma K, Kim K, Donnelly M, Young Choi H, Hyung Chang J et al. Synaptopodin protects against proteinuria by disrupting Cdc42:IRSp53:Mena signaling complexes in kidney podocytes. Am J Pathol 2007; 171: 415-427.

34. Carlier MF, Ducruix A, Pantaloni D. Signalling to actin: the Cdc42-N-WASP-Arp2/3 connection. Chem Biol 1999; 6: R235-R240.

35. Kesavan G, Sand FW, Greiner TU, Johansson JK, Kobberup S, Wu X et al. Cdc42-mediated tubulogenesis controls cell specification. Cell 2009; 139: 791-801.

36. Hong W, Guan KL. The YAP and TAZ transcription co-activators: key downstream effectors of the mammalian Hippo pathway. Semin Cell Dev Biol 2012; 23: 785-793.

37. Hong L, Kenney SR, Phillips GK, Simpson D, Schroeder CE, Noth J et al. Characterization of a Cdc42 protein inhibitor and its use as a molecular probe. J Biol Chem 2013; 288: $8531-8543$.

38. Zhang $Y, X i a ~ H, G e X$, Chen $Q$, Yuan $D$, Chen $Q$ et al. CD44 acts through RhoA to regulate YAP signaling. Cellular signalling 2014; 26: 2504-2513.

39. Mundel P, Reiser J, Zuniga Mejia Borja A, Pavenstadt H, Davidson GR, Kriz W et al. Rearrangements of the cytoskeleton and cell contacts induce process formation during differentiation of conditionally immortalized mouse podocyte cell lines. Exp Cell Res 1997; 236: 248-258.

40. Kilkenny C, Browne W, Cuthill IC, Emerson M, Altman DG. Animal research: reporting in vivo experiments: the ARRIVE guidelines. Br J Pharmacol 2010; 160: 1577-1579.

41. Bao H, Ge Y, Peng A, Gong R. Fine-tuning of NFkappaB by glycogen synthase kinase 3beta directs the fate of glomerular podocytes upon injury. Kidney Int 2015; 87: 1176-1190.

42. Lloberas J, Celada A. p21 (waf1/CIP1), a CDK inhibitor and a negative feedback system that controls macrophage activation. Eur J Immunol 2009; 39: 691-694.

43. Zhang Y, Liu J, Luan G, Wang X. Inhibition of the small GTPase Cdc42 in regulation of epileptic-seizure in rats. Neuroscience 2015; 289: 381-391. 
44. Zins K, Gunawardhana S, Lucas T, Abraham D, Aharinejad S. Targeting Cdc42 with the small molecule drug AZA197 suppresses primary colon cancer growth and prolongs survival in a preclinical mouse xenograft model by downregulation of PAK1 activity. J Trans/ Med 2013; 11: 295.

45. Toyonaga J, Tsuruya K, Ikeda H, Noguchi H, Yotsueda H, Fujisaki K et al. Spironolactone inhibits hyperglycemia-induced podocyte injury by attenuating ROS production. Nephrol Dial Transplant 2011; 26: 2475-2484.

46. Zhang L, Li R, Shi W, Liang X, Liu S, Ye Z et al. NFAT2 inhibitor ameliorates diabetic nephropathy and podocyte injury in db/db mice. Br J Pharmacol 2013; 170: 426-439.

47. Si J, Ge Y, Zhuang S, Wang LJ, Chen S, Gong R. Adrenocorticotropic hormone ameliorates acute kidney injury by steroidogenic-dependent and -independent mechanisms. Kidney Int 2013; 83: 635-646. (c) (i) Cell Death and Disease is an open-access journal published by Nature Publishing Group. This work is licensed under a Creative Commons Attribution 4.0 International License. The images or other third party material in this article are included in the article's Creative Commons license, unless indicated otherwise in the credit line; if the material is not included under the Creative Commons license, users will need to obtain permission from the license holder to reproduce the material. To view a copy of this license, visit http://creativecommons.org/licenses/by/4.0/ 